\title{
Conductas alimentarias de riesgo e interiorización del modelo estético corporal en participantes de certámenes de belleza
}

\author{
Risk eating behaviors and internalization of the body aesthetic model in beauty \\ pageant participants
}

\author{
Leticia S. Salazar-Molar ${ }^{a}$,Vanessa M. Vázquez-Vázquez ${ }^{b}$, Lilián E. Bosques-Brugada ${ }^{c}$
}

\begin{abstract}
:
Beauty contests have been seen as potentially favorable means of issuing beauty standards, which can be associated with the adoption of risky eating behaviors (REB) that compromise the health (physical and mental) of those who participate in these contests. Therefore, the objective of this work was to analyze the relationships between REB, body dissatisfaction, internalization of body aesthetic model and self-esteem of 133 women between 15 and 27 years old $(M=20.70, S D=2.50)$ to whom were applied: a sociodemographic survey, the Eating Attitudes Test, the Questionnaires of Body Shape and of Sociocultural Influences on the Aesthetic Body Shape Model as well as the Rosenberg Self-Esteem Scale were applied. The results of the correlation test show the existence of significant associations between all the variables evaluated, the relationship of the REB with body dissatisfaction having a greater strength $(r s=$ $.57, p<.001)$ and of the latter with the internalization of body aesthetic model $(r s=0.76, p<0.001)$. These findings show the need to consider body satisfaction as one of the preventive strategic routes of REB, as well as the close relationship that body dissatisfaction has with the internalization of body aesthetic model to which the participants of beauty contests are exposed.
\end{abstract}

Keywords:

Beauty pageants, modeling, dietary risk behaviors, body dissatisfaction, women

\section{Resumen:}

Los concursos de belleza, se han visto como medios potencialmente favorecedores de la emisión de estándares de belleza, que pueden asociarse con la adopción de conductas alimentarias de riesgo (CAR) que comprometan la salud (física y mental) de quienes son partícipes de estos certámenes. Por lo que, el objetivo del presente trabajo fue analizar las relaciones existentes entre las CAR, la insatisfacción corporal, la interiorización del modelo estético corporal y la autoestima de 133 mujeres de entre 15 y 27 años $(M=$ 20.70, DE = 2.50), a quienes se les aplicaron: un cuestionario sociodemográfico, el Eating Attitudes Test, el Body Shape Questionnaire, el cuestionario de Influencias del Modelo Estético Corporal, así como la Escala de Autoestima de Rosenberg. Los resultados de la prueba de correlación mostraron la existencia de asociaciones significativas entre la mayoría de variables evaluadas, teniendo una mayor fuerza la relación de las CAR con la insatisfacción corporal $\left(r_{s}=.57, p<.001\right)$ y de esta segunda con la interiorización del modelo estético corporal $\left(r_{s}=.76, p<.001\right)$. Estos hallazgos evidencian la necesidad de contemplar a la satisfacción corporal como una de las vías estratégicas preventivas de las CAR, al igual que la estrecha relación que guarda la insatisfacción corporal con la interiorización del modelo estético corporal de las participantes de certámenes de belleza.

\section{Palabras Clave:}

Concursos de belleza, modelaje, conductas alimentarias de riesgo, insatisfacción corporal, mujeres

Introducción
Las sociedades contemporáneas se han caracterizado por exigencias cada vez mayores en los estándares corporales [1], siendo los concursos de belleza uno de los

\footnotetext{
${ }^{a}$ Autor de Correspondencia, Universidad Autónoma del Estado de Hidalgo, Email: sa369416@uaeh.edu.mx

b Universidad Autónoma del Estado de Hidalgo, https://orcid.org/0000-0003-2357-4157, Email: vanessa_vazquez@uaeh.edu.mx

c Universidad Autónoma del Estado de Hidalgo, https://orcid.org/0000-0002-3969-683X, Email: lilian_bosques@uaeh.edu.mx
} 
medios en los que se han visto reflejadas dichas exigencias, imágenes globalizadas no sólo de propuestas de prototipos físicos deseables, sino también de etnia, raza y clase social que vinculan definiciones culturales de "belleza" y "atractivo corporal" con ideas de éxito [2]. Tales demandas podrían incidir en la alteración de los comportamientos alimentarios o incluso la adopción de patrones inadecuados en la ingesta de alimentos por parte de las personas, entre ellos: atracones, dietas restrictivas, uso de laxantes, enemas, diuréticos, vómito autoinducido, práctica excesiva de ejercicio físico, vigilancia permanente sobre el propio cuerpo, etcétera $[3,4]$. Estos comportamientos son considerados como conductas alimentarias de riesgo (CAR) al no satisfacer de manera general los criterios diagnósticos (en tanto a frecuencia, intensidad y duración) de los trastornos alimentarios y de la ingestión de alimentos (TAIA), aunque en ambos casos, todas las acciones previamente mencionadas tienen como propósito la pérdida de peso corporal y con posibles repercusiones en la salud física y mental [5].

Entre los datos epidemiológicos de los TAIA, a nivel nacional, se ha estimado contar con registros anuales de 20 mil casos de anorexia y bulimia, siendo la población de entre 15 y 19 años la de mayor riesgo [2]. Particularmente, en el caso de las participantes de concursos de belleza, Thompson et al. [6] señalaron que, el $26 \%$ de las aspirantes a reinas de belleza han sido diagnosticadas $o$ han percibido presentar un trastorno alimentario, considerando a esta población en mayor riesgo para presentar alteraciones en la ingesta de alimentos en contraste con mujeres que no hayan sido partícipes de estos concursos.

Por su parte, los factores socioculturales son considerados de gran relevancia en las CAR y posteriormente en los TAIA, en particular durante la adolescencia, debido a las características implícitas en esta etapa del desarrollo, por ejemplo, las dirigidas a la búsqueda de la identidad a través de la imitación y la comparación con modelos estéticos a los cuales aspiran $[7,8]$. En relación a ello, García-Ochoa [9] ha señalado que, los modelos publicitarios influyen en la valoración del propio cuerpo y en el intento por asemejarse a los atributos físicos presentados en estos medios. Losada et al. [10] con el objetivo de evaluar el grado de asociación entre la interiorización del modelo estético corporal y las CAR, evaluaron a una muestra de 71 adolescentes de entre 13 a 18 años de edad adscritos a escuelas secundarias de Buenos Aires. En los resultados se observó una fuerte asociación del riesgo por presentar sintomatología de TAIA con una mayor interiorización en el modelo estético corporal $(r=.70, p<.001)$.
En cuanto a la población joven, Mancilla-Díaz et al. [11] evaluaron el rol de la interiorización del modelo estético corporal sobre las conductas alimentarias de riesgo en muestras españolas (254 mujeres y 184 varones) y mexicanas (243 féminas y 181 hombres), con una edad promedio de 20 años. Los análisis comparativos por sexo mostraron que, las mujeres alcanzaron mayores puntajes en el autoreporte de la susceptibilidad a la interiorización del modelo estético corporal $(F[1,861]=4.06 ; p=.04)$, el malestar con la imagen corporal $(F[1,861]=13.77 ; p=$ .001), así como en la influencia de los mensajes verbales $(F[1,861]=4.46 ; p=.035)$ y de los modelos sociales $(F$ $[1,861]=6.97 ; p=.008)$ en contraste con los hombres. Asimismo, se encontraron diferencias estadísticamente significativas entre quienes presentaban sintomatología de TAIA de quienes no la reportaron, reconociéndose una mayor interiorización del modelo estético corporal en el primer grupo $(F[1,861]=41.27 ; p=.0001)$.

El estudio reciente de Amaya-Hernández et al. [12] en el que se evaluó a 273 preadolescentes y 175 adolescentes (de ambos sexos) con la finalidad de conocer la relación existente entre la influencia de pares, la interiorización del modelo estético corporal, la insatisfacción corporal y las conductas alimentarias anómalas o CAR, se halló en primera instancia que, la insatisfacción corporal y la interiorización del modelo estético corporal están estrechamente asociadas con las CAR $(r=.64$ y $r=.35$ en los adolescentes, así como en los preadolescentes $r$ $=.52 \mathrm{y} r=.32, p<.05)$. En tanto al modelo por ecuaciones estructurales analizados por los autores se halló que, en ambas submuestras, las CAR fueron predichas únicamente por la insatisfacción corporal $(\beta=.52$ [preadolescentes] y $\beta=.63$ [adolescentes]) y esta última variable fue impactada por la interiorización del modelo estético corporal $(\beta=.31$ [preadolescentes] y $\beta=.41$ [adolescentes]) junto con la popularidad con pares de sexo opuesto $(\beta=.58$ [preadolescentes] y $\beta=.42$ [adolescentes]).

Otra variable psicológica relacionada con las CAR ha sido la autoestima, que de acuerdo con la literatura además del sentido de autovalía, incluye la suma de juicios que una persona tiene de sí misma [13]. En este sentido, Wonderlich et al. [14] con el propósito de comparar aspectos como: autoestima, depresión, insatisfacción corporal y sintomatología de trastornos alimentarios, realizaron un estudio contando con 21 mujeres (11 participantes en concursos de belleza infantil y 11 jóvenes que no reportaron haber participado en este tipo de certámenes) dentro de un rango etario de 19 y 25 años de edad. Entre sus resultados encontraron que, las mujeres participantes de concursos de belleza infantil 
puntuaron significativamente más alto en las medidas de insatisfacción corporal $(F=4.99, \mathrm{p}=.04)$, desconfianza interpersonal $(F=4.90, \mathrm{p}=.04)$ y desregulación de los impulsos $(F=6.92, p=.02)$ en comparación con las mujeres que no participaron en dichos concursos. Everhart [15] por su parte, investigó las diferencias en tanto a la insatisfacción corporal, la depresión y la autoestima en 20 participantes de concursos de belleza ( $M=21$ años) y 20 que nunca habían sido parte de estos certámenes ( $\mathrm{M}=32$ años). El análisis de resultados confirmó únicamente diferencias significativas en las variables: imagen corporal ideal, insatisfacción corporal, así como autoestima $(t=3.69,3.69$ y 3.50, $p<.001$, respectivamente). Las participantes de certámenes obtuvieron mayores puntajes en: imagen corporal ideal $(M=18.5, D E=13.48)$, insatisfacción corporal $(M=17.5$, $D E=10.20)$ y autoestima $(\mathrm{M}=12.9, D E=10.41)$ en comparación a las jóvenes no participantes $(\mathrm{M}=33, D E$ = 11.29; $\mathrm{M}=8, D E=6.96 ; \mathrm{y} \mathrm{M}=28.1, D E=16.37$, respectivamente). Lo que significó que, en mayor medida las mujeres del primer grupo consideraban como ideal un cuerpo con medidas más pequeñas, tener una menor satisfacción corporal, aunque con aparente mayor autoestima. Asimismo, este autor afirmó asociaciones estadísticamente significativas entre la insatisfacción corporal y la autoestima en ambas submuestras $(r=.49$, $r=.39, p<.05$, respectivamente en participantes concursantes y no de certámenes de belleza).

De manera similar, Thompson y Hammond [16] realizaron un estudio donde se examinó la autoestima, la insatisfacción corporal, la dieta y la preocupación por la imagen corporal en 131 mujeres que habían sido concursantes de certámenes de belleza ( $M=26$ años). En los resultados se reportó que, el $26 \%$ de las participantes reconocieron un trastorno alimentario, y reportaron que esta problemática surgió al momento o después de participar en el certamen de belleza; el $48 \%$ manifestó desear mayor delgadez y el $57 \%$ estar a dieta para perder peso. De acuerdo con lo reportado por los autores, se halló evidencia de una asociación negativa entre la autoestima y la preocupación por la imagen corporal $(r=-.55, p<.05)$, además de relaciones entre el nivel alcanzado por la participante en los certámenes con la autoestima $(r=.27, p<.05)$ y el número de certámenes en los que participó y fue finalista con el nivel alcanzado en los certámenes $(r=.31, p<.05)$. A partir de lo mencionado anteriormente, se realza la importancia de estudiar la asociación de las variables mencionadas en las jóvenes concursantes de certámenes de belleza. Por lo que la presente investigación buscó analizar la relación entre las conductas alimentarias de riesgo en participantes de certámenes de belleza con insatisfacción corporal, interiorización del modelo estético corporal de la delgadez y autoestima.

\section{Método}

\section{Participantes}

Se realizó un muestreo no probabilístico por conveniencia. Las participantes del presente estudio fueron 133 mujeres mexicanas (adolescentes y jóvenes) de entre los 15 y 27 años, con una edad promedio de $20.70(D E=2.50)$, que habían formado parte de concursos de belleza "estándar" en diferentes niveles (municipales, estatales, nacionales e internacionales). La mayoría de las participantes $(77.4 \% ; n=103)$ refirieron contar con una escolaridad de licenciatura (entre 15-18 años de estudios), seguidas de las concursantes de nivel bachillerato (entre 11-14 años de estudios) el 15.8\% ( $n=$ 21), y nueve jóvenes estudiantes de posgrado (18 o más años de estudios, representando un $6.8 \% ; n=9$ ).

\section{Tipo de estudio}

El presente estudio fue de naturaleza cuantitativo de tipo no experimental-transversal con un alcance correlacional.

\section{Instrumentos}

Eating Attitudes Test-26 (EAT-26). Instrumento diseñado por Garner et al. [17] para la detección de síntomas característicos de anorexia nerviosa, compuesto por 26 reactivos con una escala de respuesta tipo Likert con seis opciones de respuesta (de nunca $=1$ a siempre $=6$ ), el cual mostró una confiabilidad interna adecuada en muestras clínicas y no clínicas $(\alpha=.90$ y .83, respectivamente). En México Franco-Paredes et al. [18] corroboraron también una consistencia interna similar $(\alpha=.83)$ en una muestra de mujeres.

Body Shape Questionnaire-34 (BSQ-34). EI BSQ-34 fue creado por Cooper et al. [19], dirigido para explorar la presencia de insatisfacción corporal, a través de 34 ítems con una escala de respuesta tipo Likert de seis puntos (que van de nunca $=1$ a siempre $=6$ ). Esta escala fue validada en México por Vázquez et al. [20], en una muestra de 472 mujeres (incluyendo muestra clínica), con un rango de edad de 13 a 30 años. El instrumento quedó conformado por sus 34 reactivos originales que, de acuerdo con el análisis factorial quedaron agrupados en dos dimensiones: Malestar corporal normativo $(\alpha=.95)$ y Malestar corporal patológico $(\alpha=.94)$ reportando una confiabilidad interna adecuada de la escala global $(\alpha=$ $.98)$, con un porcentaje de varianza explicada total de $63.83 \%$. En tanto a la validez discriminante, el BSQ-34 cuenta con una adecuada capacidad para diferenciar personas con anorexia, bulimia y población no clínica $(\lambda$ 
de Wilks $=.485, \times 2(g l=1)=278.830, p \leq .001)$. Mientras que, el punto de corte reportado para las mujeres mexicanas fue de 110 puntos.

\section{Cuestionario de Influencias del Modelo Estético} Corporal-40 (CIMEC-40). Este instrumento fue creado por Toro et al. [21], con la finalidad de medir las influencias culturales relevantes que contribuyen a provocar, facilitar o justificar el adelgazamiento por razones estéticas y sociales, a través de 40 reactivos con una escala de respuesta tipo Likert de tres puntos (de no nunca $=0$ a sí siempre $=2$ ). La adaptación y validación del CIMEC-40 en mujeres mexicanas de entre 14 y 33 años fue realizada por Vázquez et al. [22] quedando configurado por 35 ítems integrados en cuatro factores: Influencia de la Publicidad $(\alpha=.92)$, Malestar por la Imagen Corporal $(\alpha=.86)$, Influencia de los Modelos Estéticos Corporales $(\alpha=.75)$ e Influencia de las Relaciones Sociales ( $\alpha=.63$ ), con una satisfactoria consistencia interna global $(\alpha=.94)$. Dichas dimensiones reportaron tener un porcentaje total de varianza explicada del $45.3 \%$.

Escala de Autoestima de Rosenberg (EAR) [23]. Este instrumento tiene como objetivo explorar los sentimientos de valía personal y de respeto a sí mismo, a través de 10 reactivos con una escala de respuesta tipo Likert de cuatro puntos (que va desde muy de acuerdo = 1 a muy en desacuerdo = 4). En México el instrumento ha sido validado por Jurado et al. [24] en estudiantes de bachillerato y licenciatura de entre 14 y 49 años. Tras el análisis factorial confirmatorio [25] se reportó haber obtenido un total de ocho ítems agrupados en dos factores: Afirmaciones negativas y Afirmaciones positivas. La escala cuenta con un alpha de Cronbach de 79 .

\section{Procedimiento}

El presente estudio se llevó a cabo bajo las consideraciones éticas de código ético del psicólogo [26]. A las mujeres participantes se les aplicó una batería de pruebas (previo consentimiento informado), integrada por los cuatro instrumentos psicométricos descritos previamente. La evaluación se llevó a cabo entre febrero y marzo del 2020 , en una sola sesión con una duración de aproximadamente 50 minutos.

\section{Procedimiento}

\section{Análisis estadísticos}

Los datos fueron analizados en el Paquete Estadístico para las Ciencias Sociales (SPSS por sus siglas en inglés, versión 25), donde se llevaron a cabo los análisis descriptivos. Además, para examinar la distribución de los datos de las variables de estudio, se realizó el análisis de normalidad de los diferentes instrumentos (EAT-26, $B S Q-34, C I M E C-40$ y EAR) por medio de la prueba de Kolmogorov-Smirnov y se rechazó el supuesto de la misma en la muestra $\left(X^{2}=.12, .16, .11\right.$ y .09 , respectivamente, $p<.01)$. En este sentido, se hizo uso de la estadística no paramétrica para correlaciones con el coeficiente de Spearman $\left(r_{s}\right)$.

\section{Resultados}

De las 133 mujeres participantes de certámenes de belleza evaluadas, en su mayoría se trató de jóvenes con 20 o más años de edad (70.68\%), seguidas de las situadas aún en la etapa de adolescencia de entre 15 a 19 años (29.32\%). Las medidas de tendencia central obtenidas en las variables psicológicas medidas se presentan a continuación (ver Tabla 2):

Tabla 2. Medianas de las variables psicológicas

\begin{tabular}{lc}
\multicolumn{1}{c}{ Variable } & Md $(R Q)$ \\
\hline 1. Conductas alimentarias de riesgo & $50(30)$ \\
2. Insatisfacción corporal & $68(59)$ \\
3. Interiorización del modelo estético & $13(18.5)$ \\
corporal de la delgadez & \\
4. Autoestima & $28(13.5)$
\end{tabular}

De los instrumentos aplicados, el BSQ-34 es el único que cuenta con punto de corte establecido para población mexicana $(\geq 110)$, que en el caso del presente estudio, un $20.3 \%$ ( $n=27$ ) de las participantes de certámenes de belleza superó dicho punto de corte, es decir, presentaron un nivel relevante de insatisfacción corporal.

El objetivo general del presente estudio fue analizar la relación entre las conductas alimentarias de riesgo en participantes de certámenes de belleza con la insatisfacción corporal, la interiorización del modelo estético corporal de la delgadez y la autoestima. Los resultados hallados mostraron asociaciones significativas entre las CAR con la totalidad de variables evaluadas (ver Tabla 1), es decir a mayor presencia de conductas alimentarias de riesgo se identificó una mayor insatisfacción corporal, interiorización del modelo estético corporal y menor autoestima $\left(r_{s}=.57, .48\right.$ y -.31 respectivamente, $p=.001)$. Asimismo, se halló relacionada estrechamente la insatisfacción corporal con la interiorización del modelo estético corporal $\left(r_{s}=.76, p\right.$ $=.001)$. 
Tabla 1.

Correlaciones entre CAR, insatisfacción corporal, interiorización del modelo estético corporal y autoestima en mujeres participantes de certámenes de belleza $(n=133)$

\begin{tabular}{|c|c|c|c|c|}
\hline Variable & 1 & 2 & 3 & 4 \\
\hline $\begin{array}{l}\text { 1. Conductas alimentarias } \\
\text { de riesgo }\end{array}$ & 一 & $.57^{\star}$ & $.48^{\star}$ & $-.31^{\star}$ \\
\hline 2. Insatisfacción corporal & - & - & $.76^{\star}$ & -.13 \\
\hline $\begin{array}{l}\text { 3. Interiorización del modelo } \\
\text { estético corporal de la } \\
\text { delgadez } \\
\text { 4. Autoestima }\end{array}$ & 一 & - & 一 & -.04 \\
\hline
\end{tabular}

Nota: * $p<.001$. Elaboración propia.

\section{Discusión y conclusiones}

El objetivo general del presente trabajo fue analizar las asociaciones de las conductas alimentarias de riesgo (atracones, dietas restrictivas, uso de laxantes, diuréticos, enemas, entre otros comportamientos) con la insatisfacción corporal, la interiorización del modelo estético corporal y la autoestima de mujeres participantes de concursos de belleza. Los resultados hallados, aportan evidencia de la interacción significativa de las variables evaluadas.

En lo que concierne a la insatisfacción corporal, se identificó como un factor vinculado estrechamente con las CAR; es decir una mayor inconformidad con la imagen corporal y la búsqueda de cambios en la misma está emparentada con mayores conductas alimentarias inadecuadas para lograr dicho fin, lo cual es congruente con lo reportado por Amaya-Hernández et al. [12], incluso al reportar a la insatisfacción corporal como una variable predictora de las CAR. De la misma manera las conductas alimentarias de riesgo se vieron asociadas con la interiorización del modelo estético corporal que tiene que ver con la búsqueda de estándares de belleza aceptados socialmente, resultados congruentes con Amaya-Hernández et al. [12], Losada et al. [10] y Mancilla-Díaz et al. [11] debido al rol que se ha visto que tiene la influencia de las expectativas sociales en la adopción de conductas que buscan la pérdida de peso. Igualmente, estas conductas insanas se vieron relacionadas con una menor autoestima en concurrencia con lo reportado recientemente en el metanálisis de Colmsee et al. [27] en el que se atañe a la baja autoestima en un rol de factor de riesgo para los diferentes trastornos alimentarios. Sin embargo, hay que reconocer que (contrario a lo inicialmente pronosticado), el factor autoestima fue el que obtuvo menores magnitudes de correlación con los otros factores evaluados.
A partir de los hallazgos reportados en el presente estudio, se considera pertinente ahondar en el rol de la autoestima, para dar próximo seguimiento, se podrían ejecutar modelos predictivos con variables intervinientes donde se incorporen al factor de cosificación así como otras dimensiones del autoconcepto, en la población juvenil inmersa en certámenes de belleza. Además, se reconocen como limitantes que, si bien se contemplaron en la muestra de estudio tanto adolescentes como jóvenes, no fue posible contrastar los resultados obtenidos en función de esta característica, debido a que no eran proporcionales ambos grupos. Por otra parte, la segunda limitante fue la naturaleza correlacional utilizada (análisis no paramétricos), puesto que implican limitaciones en el alcance de los hallazgos reportados. Por lo que, para futuras investigaciones se sugiere la consideración de un tamaño de muestra más amplio que dé cabida a análisis comparativos en función de la etapa de vida (adolescencia-juventud) así como del tiempo que tengan las participantes en ser parte de estos concursos además de la contemplación de estudios mixtos, que permitan explorar y ampliar cualitativamente la información al respecto.

A manera de conclusión se encontró que, en una muestra de concursantes de certámenes de belleza mexicanas (adolescentes y jóvenes), a mayor nivel reportado de conductas alimentarias de riesgo, mayor grado de insatisfacción con su imagen corporal, mayor interiorización del modelo estético corporal pro-delgadez y (en una menor magnitud), menor autoestima.

\section{Referencias}

[1] Vigarelo G. Corregir el cuerpo. Historia de un poder pedagógico. Buenos Aires: Nueva Visión; 2005.

[2] Valcuende del Río J, Vásquez P. Orden corporal y representaciones raciales de clase y género en la ciudad de Cuenca (Ecuador). Chungara, Revista de Antropología Chilena. 2016; 48(2):307-317

[3] Unikel C, Díaz de León C, Rivera JA. Conductas alimentarias de riesgo y factores de riesgo asociados: desarrollo y validación de instrumentos de medición. México: Universidad autónoma metropolitana; 2017.

[4] Bolívar IJ. Reinados de belleza y nacionalización de las sociedades latinoamericanas. Iconos. Revista de Ciencias Sociales. 2007;(28): 7180.

[5] Unikel C, Díaz de León-Vázquez C, González-Forteza C, Wagner F, Rivera JA. Conducta alimentaria de riesgo, síntomas depresivos y correlatos psicosociales en estudiantes universitarios de primer ingreso. Acta Universitaria. 2015; 25(2):35-39.

[6] Thompson JK, Heinberg LJ, Altabe M N, Tantleff-Dunn, S. Exacting beauty: Theory, assessment, and treatment of body image disturbance. American Psychological Association.1999.

[7] Johnston JE ¿Por qué no me gustó? como dejar de preocuparnos por nuestro aspecto físico. Barcelona: Paidós; 1996.

[8] Carrillo-Durán MV. La transmisión de valores sociales por medio de las imágenes. La identificación de la mujer delgada igual a mujer 
triunfadora en la población adolescente. Comunicación y Sociedad 2013; XVI (2): 33.37.

[9] García-Ochoa Y. El cuerpo femenino en la publicidad. Revista ICONO 14. Revista Científica De Comunicación y Tecnologías Emergentes. 2010; 8(3): 223-243

[10]Losada A, Leonardelli E, Magliola M.. Influencia sociocultural y los trastornos de la conducta alimentaria en adolescentes. Revista Electrónica de Psicología Iztacala. 2015; 18(1): 380-410.

[11] Mancilla-Díaz JM, Lameiras-Fernández M, Vázquez-Arévalo R, Álvarez-Rayón G, Franco-Paredes K., López-Aguilar X, Tellez-Girón, MTO. Influencias socioculturales y conductas alimentarias no saludables en hombres y mujeres de España y México. Revista mexicana de trastornos alimentarios.2010; 1(1): 36-47.

[12] Amaya-Hernández A, Álvarez-Rayón G, Ortega-Luyando M, Mancilla-Díaz, J M. Peer influence in preadolescents and adolescents: A predictor of body dissatisfaction and disordered eating behaviors: Revista Mexicana de Trastornos Alimentarios. 2017; 8:31-39.

[13]Proaños NM. La autoestima como factor influyente en el rendimiento académico. Colombia (Tesis de maestría).2017.

[14]Wonderlich AL, Ackard DM, Henderson JB. Chilhood beauty pagean contestants: associations with adult disorded eating and mental health, Eating Disorders: The Journal of treatment \& Prevention. 2005; 13(3): 291-301

[15] Everhart A. Entertaining a false reality: a social comparation examination of beauty pageant participation and the effect on body dissatisfaction, depression, and self-eestem. Graduate Theses, Dissertations, and Problem Reports. 2011. Tesis de posgrado.

[16] Thompson SH, Hammond K. Beauty is as beauty does: body image and self-esteem of pageant contestants. Eat Weight Disord. 2003; 8(3): 231-237.

[17]Garner, DM, Olmsted MP, Bohr Y, Garfinkel PE. The Eating Attitudes Test: Psychometric features and clinical correlates. Psychological Medicine. 1982; 12(4): 871-878.

[18]Franco-Paredes K, Solorzano M, Díaz-Reséndiz FJ, HidalgoRassmussen C. (2016). Confiabilidad y estructura factorial del Test de Actitudes Alimentarias (EAT-26) en mujeres mexicanas. Revista Mexicana de Psicología, suplemento. 2016; 278-279.

[19] Cooper P, Taylor M, Cooper Z, Fairburn C. The development and validation of the Body Shape Questionnaire. International Journal of Eating Disorders. 1987; 6(4): 485-495.

[20] Vázquez R, Galán J, López X, Álvarez G, Mancill, JM, Caballero A Unikel C. Validez del Body Shape Questionnaire (BSQ) en mujeres mexicanas. Revista Mexicana de Trastornos Alimentarios. 2011; 2(1): $42-52$

[21] Toro J, Salamero M, Martínez E. Assessment of sociocultural influences on the aesthetic body shape model in anorexia nervosa. Acta Psychiatrica Scandinavica. 1994; 89(3): 147-51.

[22] Vázquez R, Álvarez G, Mancilla JM. Consistencia interna y estructura factorial del Cuestionario de Influencia de los Modelos Estético Corporales (CIMEC), en población mexicana. Salud Mental.2000; 23(6): 18-24

[23] Rosenberg M. Society and the adolescent self-image. Princenton. Princenton University Press; 1965.

[24]Jurado D, Jurado S, López K, Querevalú B. Validez de la Escala de Autoestima de Rosenberg en universidades de la ciudad de México. Revista latinoamericana de Medicina Conductual.2015; 5(1): 18-22.

[25] Bosques LE. Efecto de la adhesión a los estereotipos de género sobre la sintomatología de trastorno del comportamiento alimentario y dismorfia muscular: Identificación de variables mediadoras. México (Tesis de doctorado). 2015
[26] Sociedad Mexicana de Psicología. Código ético del psicólogo. 4a ed. México: Trillas; 2009.

[27] Colmsee I. S. 0, Hank P, Hank P, Bosnjak M. Low self-esteem as a risk factor for eating disorders: a meta-analysis. Zeitschriff fur Psychologie; 229(1): 48-69. 